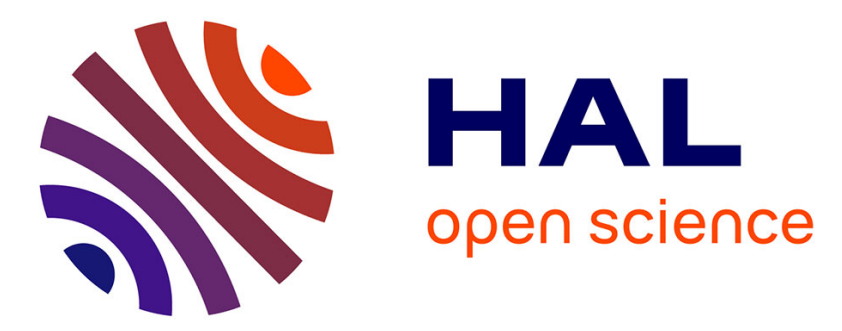

\title{
Exploring the shear strain contribution to the uniaxial magnetic anisotropy of (Ga,Mn)As
}

M. Kraimia, L. Largeau, K. Boujdaria, B. Croset, C. Mocuta, A. Lemaitre, C. Gourdon, L. Thevenard

\section{- To cite this version:}

M. Kraimia, L. Largeau, K. Boujdaria, B. Croset, C. Mocuta, et al.. Exploring the shear strain contribution to the uniaxial magnetic anisotropy of (Ga,Mn)As. Journal of Applied Physics, 2020, 127 (9), pp.093901. 10.1063/1.5140078 . hal-02475068v2

HAL Id: hal-02475068

https://hal.science/hal-02475068v2

Submitted on 6 Mar 2020

HAL is a multi-disciplinary open access archive for the deposit and dissemination of scientific research documents, whether they are published or not. The documents may come from teaching and research institutions in France or abroad, or from public or private research centers.
L'archive ouverte pluridisciplinaire HAL, est destinée au dépôt et à la diffusion de documents scientifiques de niveau recherche, publiés ou non, émanant des établissements d'enseignement et de recherche français ou étrangers, des laboratoires publics ou privés. 


\section{Exploring the shear strain contribution to the uniaxial magnetic anisotropy of (Ga,Mn)As}

Cite as: J. Appl. Phys. 127, 093901 (2020); https://doi.org/10.1063/1.5140078

Submitted: 26 November 2019 . Accepted: 18 February 2020 . Published Online: 05 March 2020

M. Kraimia, L. Largeau (D), K. Boujdaria (D), B. Croset (D), C. Mocuta (D), A. Lemaître (D), C. Gourdon (D), and L. Thevenard (i)

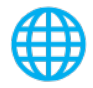

Lock-in Amplifiers Find out more today

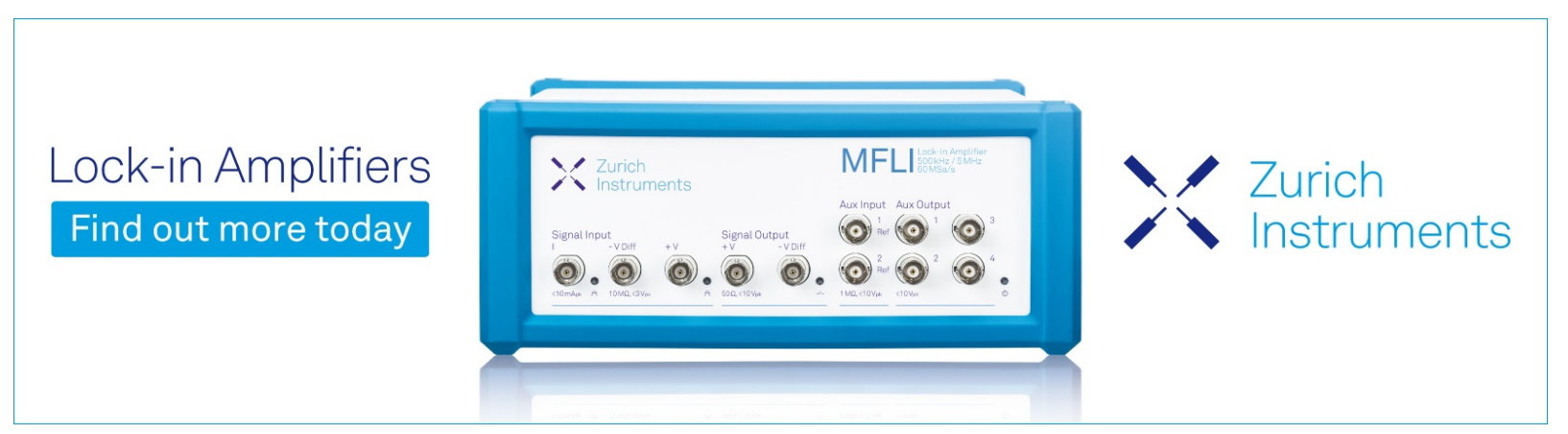

Zurich

Instruments 


\title{
Exploring the shear strain contribution to the uniaxial magnetic anisotropy of (Ga,Mn)As
}

\author{
Cite as: J. Appl. Phys. 127, 093901 (2020); doi: 10.1063/1.5140078 \\ Submitted: 26 November 2019 . Accepted: 18 February 2020 . \\ Published Online: 5 March 2020
}

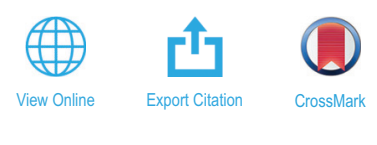

M. Kraimia, ${ }^{1,2}$ L. Largeau, $^{3}$ (D) K. Boujdaria, ${ }^{2}$ (D) B. Croset, ${ }^{1}$ (D) C. Mocuta, ${ }^{4}$ (D) A. Lemaître, ${ }^{3}$ (iD C. Gourdon, ${ }^{1}$ (D) and L. Thevenard ${ }^{1, a)}$ (iD

\begin{abstract}
AFFILIATIONS
${ }^{1}$ CNRS, Institut des Nanosciences de Paris, Sorbonne Université, 4 place Jussieu, 75252 Paris, France

${ }^{2}$ Faculté des Sciences de Bizerte, LRO1ES15 Laboratoire de Physique des Matériaux: Structure et Propriétés, Université de Carthage, 7021 Zarzouna, Bizerte, Tunisia

${ }^{3}$ Centre de Nanosciences et de Nanotechnologies, CNRS, Univ. Paris-Sud, Université Paris-Saclay, 91120 Palaiseau, France

${ }^{4}$ Synchrotron SOLEIL, L'Orme des Merisiers Saint-Aubin, F-91192 GIF-sur-YVETTE CEDEX, France
\end{abstract}

a) Author to whom correspondence should be addressed: thevenard@insp.jussieu.fr

\begin{abstract}
We present an experimental and $\mathbf{k} \cdot \mathbf{p}$ theoretical study on the origin of the strong in-plane uniaxial magnetic anisotropy in (Ga,Mn)As layers, unexpected from the cubic crystalline structure. The symmetry lowering can be accounted for by structural or effective shear strains. We find theoretically out-of-plane and in-plane magnetic anisotropy constants being linear with the shear strain. Searching for a real shear strain arising from lattice relaxation, we perform two types of measurements: anomalous x-ray diffraction and strain-induced optical birefringence, at room temperature. Working on a strongly anisotropic (Ga,Mn)As layer, the estimated $\epsilon_{x y}=10^{-4}$ was not found although it lied an order of magnitude above the detection threshold. This ensemble of results indicates as unlikely a relaxation-driven uniaxial anisotropy. As previously suggested theoretically, the magnetic symmetry-lowering could instead originate from the anisotropic incorporation of $\mathrm{Mn}$ atoms during growth. This would yield a perfectly in-plane matched lattice, with an anisotropy that could nevertheless be modeled as an effective shear strain and modified by an external shear stress, in agreement with the existing experimental literature.
\end{abstract}

Published under license by AIP Publishing. https://doi.org/10.1063/1.5140078

\section{INTRODUCTION}

Uniaxial anisotropy in ferromagnetic layers, tracks, and nanostructures is a key factor for technological applications such as the straightforward encoding of " 0 " and " 1 " bits for memory and storage devices. Materials with out-of-plane (OP) uniaxial anisotropy have, for instance, been used to increase storage density in perpendicular magnetic recording. ${ }^{1}$ In various schemes for noninductive magnetization manipulation and switching, in-plane (IP) uniaxial anisotropy offers an alternate and versatile option for fixing the magnetization easy axis, avoiding reliance only on shape anisotropy. $^{2-}$

Whereas OP uniaxial anisotropy is fairly well understood, 5,6 much less is known about the origin of IP uniaxial anisotropy. It can arise, for instance, from an interfacial effect, ${ }^{7}$ anisotropic grain domains, ${ }^{8}$ or anisotropic relaxation of the epitaxial strain. ${ }^{8,9}$ It can be obtained by molecular beam epitaxy such as in $\mathrm{Fe} / \mathrm{GaAs}^{10-12}$ and the semiconductor (Ga,Mn)As, ${ }^{6,13-19}$ or with better control by various other growth techniques.

In layers of $(\mathrm{Ga}, \mathrm{Mn}) \mathrm{As}_{1-y} \mathrm{P}_{y}$, the microscopic origin of an IP uniaxial anisotropy constant $K_{2} \|$ has been the subject of many experimental and theoretical investigations. ${ }^{6,13,19,22-25}$ From symmetry considerations and band theory calculation in the $\mathbf{k} \cdot \mathbf{p}$ approximation, it was soon recognized that an $\epsilon_{x y}$ strain, which may result from different strains along the [110] and [-110] directions, could explain this magnetic anisotropy and its dependence with the carrier density and temperature. ${ }^{6,18}$ The value of $\epsilon_{x y} \approx 1-5 \times 10^{-4}$ obtained from these calculations was reasonably small, lower than the epitaxial strain by one order of magnitude. It has been the subject of a long-standing debate, not yet settled, whether the $\epsilon_{x y}$ strain is a real strain, or the parameter of a perturbation Hamiltonian lowering the symmetry of the zinc blende crystal.

Experimental results have repeatedly evidenced the modification of the IP uniaxial anisotropy via strain, e.g., using a piezoelectric 
transducer to apply stress, ${ }^{22}$ or deep etching of narrow bars to relax the epitaxial strain anisotropically. ${ }^{14}$ It has also been shown that thin $(\mathrm{Ga}, \mathrm{Mn})$ As bars oriented along the [110], [100], and [-110] directions relax differently, leading to varying magnetic anisotropies. ${ }^{16} \mathrm{~A}$ shear strain-dependence of the anisotropy also seems necessary to account for surface acoustic wave (SAW) driven magnetization excitation and switching in IP magnetized $(\mathrm{Ga}, \mathrm{Mn})$ As through the magnetoelastic interaction. ${ }^{26,27}$ The microscopic origin of this IP symmetry breaking would, for instance, be the [110]/[-110] anisotropic incorporation of $\mathrm{Mn}$ atoms due to stacking faults, ${ }^{25}$ or $\mathrm{Mn}$ dimer formation $^{13,23}$ due to GaAs surface reconstruction. ${ }^{12,28,29}$ This could either lead to a genuine IP strain relaxation, potentially detectable experimentally, or to a built-in shear stress, which would be virtually undetectable once the layer resting in perfect epitaxy onto its substrate.

Another approach is to consider that, from a macroscopic point of view, any depth dependence of the magnetic properties could lower the crystal symmetry from $D_{2 d}$ to $C_{2 v}$ hence making the [110] and [-110] directions non-equivalent. However, there is no clear systematic experimental evidence of such depth dependence. ${ }^{9,15,30-32}$ The dissymmetry of the two interfaces has also been invoked, since the existence of a spin-orbit field arising from the Rashba inversion asymmetry term ${ }^{33,34}$ or the Dzyaloshinskii-Moriya interaction ${ }^{35}$ reveals the breaking of the $z$-symmetry. Yet, a pure interfacial origin of the IP uniaxial magnetic anisotropy was ruled out by a thickness-dependence study. ${ }^{15}$

In this report, we investigate theoretically and experimentally the shear-strain origin of IP anisotropy in (Ga,Mn)As layers. While the few reports of $\mathrm{x}$-ray diffraction on such samples have not shown any difference of the lattice parameter along [110] and $[-110],{ }^{15,25}$ we renew the attempt to reveal a static $\epsilon_{x y}$ shear strain by working on thinner and more magnetically anisotropic layers, and combining different experimental techniques. Using the band structure obtained from the $\mathbf{k} \cdot \mathbf{p}$, exchange and strain Hamiltonians, we first determine the parameters (exchange, carrier density, and static shear strain) consistent with the measured magnetic anisotropy constants. Two very different experiments are then shown, aiming to reveal the presence of the estimated $10^{-4}$ shear strain: direct observation of the in-plane lattice by $\mathrm{x}$-ray diffraction and photoelasticity-induced optical birefringence. This ensemble of results points to an upper boundary of a few $10^{-5}$ for any genuine shear strain. This rules out anisotropic IP strain relaxation as the main origin of the uniaxial anisotropy in $(\mathrm{Ga}, \mathrm{Mn})$ As and favors alternative scenarios instead.

\section{EXPERIMENTAL UNIAXIAL ANISOTROPIES}

\section{Correlation between OP and IP anisotropy constants}

Phenomenologically, the magnetic free energy in the absence of applied magnetic field is defined as ${ }^{36}$

$$
\begin{aligned}
E_{m}= & -K_{2 \perp} \cos ^{2} \theta-K_{2 \|} \sin ^{2} \theta \sin ^{2}\left(\phi-\frac{\pi}{4}\right) \\
& -\frac{K_{4 \|}}{8} \sin ^{4} \theta(3+\cos (4 \phi))-\frac{K_{4 \perp}}{2} \cos ^{4} \theta-\frac{\mu_{0}}{2} M_{S}^{2} \sin ^{2} \theta,
\end{aligned}
$$

where $K_{2 \perp}$ and $K_{2 \|}$ are the uniaxial OP and IP anisotropy constants, respectively, and $K_{4 \|}$ and $K_{4 \perp}$ are the cubic anisotropy constants.

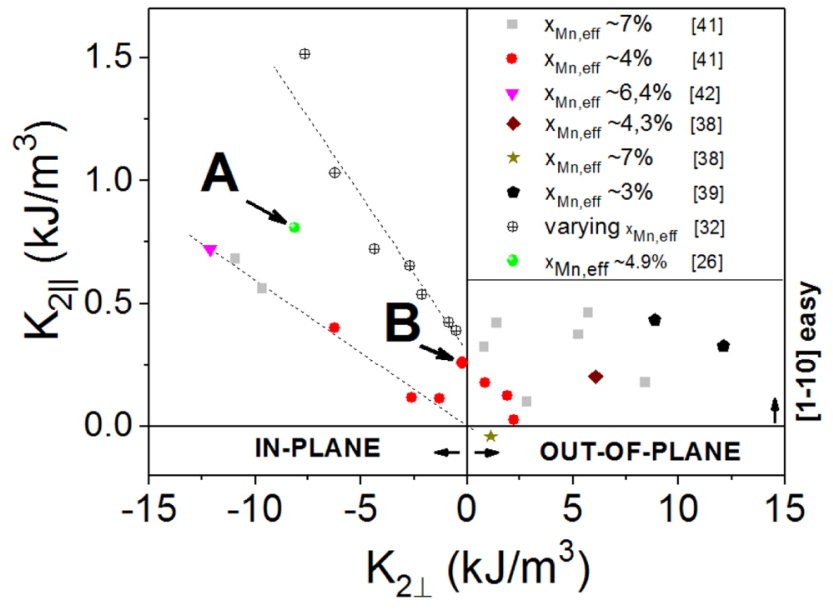

FIG. 1. Experimental uniaxial IP anisotropy constants $K_{2}$ vs uniaxial OP anisotropy constant $K_{2 \perp}$ for several sets of $(\mathrm{Ga}, \mathrm{Mn}) \mathrm{As}^{26,32,38,39}$ and $(\mathrm{Ga}, \mathrm{Mn})$ $(A s, P)^{38-42}$ epitaxial layers at low temperatures. Dashed lines are guides for the eyes. All layers are $50 \mathrm{~nm}$ thick except in Refs. $42(200 \mathrm{~nm})$ and $32(25 \mathrm{~nm})$. Samples from Ref. 32 were highly optimized (choice of growth temperature and long anneal times in particular). Samples from Refs. 38-41 underwent a standard $1 \mathrm{~h} / 250^{\circ} \mathrm{C}$ anneal, a somewhat intermediate anneal of $16 \mathrm{~h} / 200^{\circ} \mathrm{C}$.

The last term represents the shape anisotropy and depends on the saturation magnetization $M_{s}$. Angles $\phi$ and $\theta$ are counted with respect to [100] IP and [001] OP axes, respectively.

Putting together experimental OP and IP anisotropy constants of numerous $(\mathrm{Ga}, \mathrm{Mn}) \mathrm{As}$ and $(\mathrm{Ga}, \mathrm{Mn})(\mathrm{As}, \mathrm{P})$ samples, one notices that $K_{2 \|}$ is roughly proportional to $K_{2 \perp}$ for compressively strained samples (Fig. 1). This seems unrelated to the effective concentration of $\mathrm{Mn}$ atoms, the presence of phosphorus or the layer thickness. The slope of $K_{2 \|}$ vs $K_{2 \perp}$ itself seems linked to the degree of optimization of the annealing step, as clearly comes out when comparing the data of Ref. 32 with the others. ${ }^{37}$ For tensile layers however, OP and IP anisotropies seem uncorrelated, but [1-10] remains the easier IP axis, like for the compressed samples. Since the OP anisotropy varies linearly with the lattice mismatch $(\mathrm{lm})$ between the layer and the substrate, we postulate that large shear strain might appear in the case of large positive $\mathrm{lm}$ owing to anisotropic strain relaxation. Therefore, we investigate the origin of the IP uniaxial anisotropy in two samples of similar Mn concentration, one with strong IP uniaxial anisotropy (sample A) and the other with biaxial anisotropy (sample B).

\section{Samples}

In the following, we detail the characteristics of the two samples, both grown by molecular beam epitaxy on a (001) GaAs substrate topped with a high growth temperature $\left(600^{\circ} \mathrm{C}\right) \approx 500 \mathrm{~nm}$ GaAs buffer. Sample A consists of a $(\mathrm{Ga}, \mathrm{Mn})$ As layer of thickness $45 \mathrm{~nm}$ with an effective Mn concentration $x_{\text {eff }}=5 \%$. It was annealed for $16 \mathrm{~h}$ at $200^{\circ} \mathrm{C}$. The Curie temperature is $120 \mathrm{~K}$ and $M_{s}$ is $51.3 \mathrm{kA} \mathrm{m}^{-1}$ at $T=4 \mathrm{~K}$. The strained lattice mismatch after the anneal is $\mathrm{lm}=3860 \mathrm{ppm}$, resulting in a biaxial epitaxial compressive 
in-plane strain $\epsilon_{x x}=\epsilon_{y y}=-2 \times 10^{-3}$. The magnetic anisotropy constants obtained by ferromagnetic resonance (FMR) at $T=4 \mathrm{~K}$ are $K_{2 \perp}=-8.5 \mathrm{~kJ} \mathrm{~m}^{-3}, K_{2 \|}=1 \mathrm{~kJ} \mathrm{~m}^{-3}$, and $K_{4 \|}=0.41 \mathrm{~kJ} \mathrm{~m}^{-3}$. The easy magnetic axis is IP along the [-110] direction. In this sample, magnetoelastic coupling has been well established from SAW-induced magneto-acoustic resonance and magnetization switching. ${ }^{26,27}$ A model using the $B_{2}$ magnetoelastic constant (see further in the article) obtained from the experimental value of $K_{2 \|}$ and the value of $\epsilon_{x y}$ estimated from the $\mathbf{k} \cdot \mathbf{p}$ calculation quantitatively accounted for the amplitude of SAW-induced magnetization precession, suggesting the magnetoelastic origin of $K_{2 \|}$.

Sample B is a $(\mathrm{Ga}, \mathrm{Mn}) \mathrm{As}_{0.95} \mathrm{P}_{0.04}$ layer of thickness $50 \mathrm{~nm}$ with $x_{\text {eff }}=4 \%$. It was annealed for $1 \mathrm{~h}$ at $250^{\circ} \mathrm{C}$. The Curie temperature is $85 \mathrm{~K}$. At $T=4 \mathrm{~K}$, the saturation magnetization is $40.7 \mathrm{kA} \mathrm{m}^{-1}$, the anisotropy constants are $K_{2 \perp}=-0.22 \mathrm{~kJ} \mathrm{~m}^{-3}$, $K_{2 \|}=0.26 \mathrm{~kJ} \mathrm{~m}^{-3}$, and $K_{4 \|}=1.57 \mathrm{~kJ} \mathrm{~m}^{-3}$. The strained lattice mismatch is $\operatorname{lm}=-1200 \mathrm{ppm}\left(\epsilon_{x x}=-6 \times 10^{-4}\right)$. The OP anisotropy resulting from the small tensile strain is weaker than the shape anisotropy, resulting in IP magnetization with biaxial magnetic anisotropy and easy axes close to the $\langle 100\rangle$ IP directions since $K_{2 \|} \ll K_{4 \|}$. In this sample, easy triggering of spin wave excitation by optical pulses was demonstrated. ${ }^{4}$

In the following section, we will use the lattice mismatch obtained at room temperature in order to model the band structure and magnetic uniaxial anisotropies in the low-temperature limit. This procedure, used by many authors without questioning, is actually justified by the identical temperature dependence of the perpendicular lattice parameters of $(\mathrm{Ga}, \mathrm{Mn}) \mathrm{As}$ and GaAs. ${ }^{44}$

\section{MODELING UNIAXIAL ANISOTROPIES \\ Methodology}

The magnetic anisotropy properties are studied in the framework of the effective mass approximation by using the following total Hamiltonian: $\mathcal{H}=\mathcal{H}_{\mathrm{kp}}+\mathcal{H}_{\text {exc }}+\mathcal{H}_{S}$. $\mathcal{H}_{\mathrm{kp}}$ is the six-band Kohn-Luttinger Hamiltonian, ${ }^{45}$ which was shown to be sufficient for $(\mathrm{Ga}, \mathrm{Mn})$ As for a Fermi energy up to $\simeq 300 \mathrm{meV}{ }^{46}$ We employ GaAs values for the Luttinger parameters. ${ }^{47} \mathcal{H}_{\text {exc }}$ describes the effective exchange interaction between the delocalized hole spin $s$ and the localized Mn spin $S\left(S=\frac{5}{2}\right)$ arising from $p-d$ hybridization. ${ }^{48,49}$ In the mean field approximation in which the virtual crystal approximation is made, ${ }^{49}$ one can write the exchange interaction as $\left(-x_{\text {eff }} N_{0} \beta_{p d}\langle S\rangle s\right) . N_{0}$ is the cation site concentration, $\beta_{p d}$ is the $p-d$ exchange integral. $x_{\text {eff }}$ is the effective Mn concentration which is deduced from the low-temperature saturation magnetization $M_{s}$ by $x_{\text {eff }}=\frac{M_{s}}{N_{0} S g \mu_{B}}$, where $g$ is the Landé factor and $\mu_{B}$ is the Bohr magneton. The strain effects are modeled by the Bir-Pikus Hamiltonian $\mathcal{H}_{S}{ }^{50}{ }^{5}$ Formally, this strain Hamiltonian has the same structure as the Kohn-Luttinger Hamiltonian, in which $k_{\alpha} k_{\beta}$ is replaced by $\epsilon_{\alpha \beta}$, and the Luttinger parameters are replaced by the deformation potentials, namely, $a_{v}, b_{v}$, and $d_{v}$ (for more details, see for example, Ref. 6). In the basis given in Refs. 51 and 52, it takes the following expression:

$$
\mathcal{H}_{S}=\left(\begin{array}{cccccc}
\left(A_{1 s}-A_{2 s}\right) & B_{s} & C_{s} & 0 & \frac{1}{\sqrt{2}} B_{s} & \sqrt{2} C_{s} \\
c c & \left(A_{1 s}+A_{2 s}\right) & 0 & C_{s} & \sqrt{2} A_{2 s} & -\sqrt{\frac{3}{2}} B_{s} \\
c c & 0 & \left(A_{1 s}+A_{2 s}\right) & -B_{s} & -\sqrt{\frac{3}{2}} B_{s}^{*} & -\sqrt{2} A_{2 s} \\
0 & c c & c c & \left(A_{1 s}-A_{2 s}\right) & -\sqrt{2} C_{s}^{*} & \frac{1}{\sqrt{2}} B_{s}^{*} \\
c c & c c & c c & c c & A_{1 s} & 0 \\
c c & c c & c c & c c & 0 & A_{1 s}
\end{array}\right),
$$

where cc denotes the complex conjugate. One writes the non-zero matrix elements of $\mathcal{H}_{S}$ as

$$
\begin{gathered}
A_{1 s}=a_{v}\left(\epsilon_{x x}+\epsilon_{y y}+\epsilon_{z z}\right), \\
A_{2 s}=\frac{b_{v}}{2}\left(\epsilon_{x x}+\epsilon_{y y}-2 \epsilon_{z z}\right), \\
B_{s}=d_{v}\left(\epsilon_{z x}-i \epsilon_{z y}\right), \\
C_{s}=\frac{\sqrt{3}}{2} b_{v}\left(\epsilon_{x x}-\epsilon_{y y}\right)-i d_{v} \epsilon_{x y} .
\end{gathered}
$$

Adopting the notation of Glunk et al. ${ }^{53}$ for the relaxed lattice parameter and strain, one can write $\epsilon_{z z}=\frac{a_{\perp}-a_{r e l}}{a_{r e l}}$. $a_{r e l}$ is the relaxed lattice parameter of a biaxially strained layer on a [001]-oriented substrate obtained as

$$
a_{r e l}=\frac{2 C_{12}}{C_{11}+2 C_{12}} a_{\|}+\frac{C_{11}}{C_{11}+2 C_{12}} a_{\perp}
$$

where $a_{\|}$and $a_{\perp}$ are the lateral and vertical lattice parameters of the layer, respectively. In our theoretical approach, (i) due to the $T_{d} \rightarrow D_{2 d}$ symmetry reduction, we consider the biaxial strain components, namely $\left(\epsilon_{x x}, \epsilon_{y y}, \epsilon_{z z}\right)$ with $\epsilon_{x x}=\epsilon_{y y}$ and $\epsilon_{x x}=-\frac{C_{11}}{2 C_{12}} \epsilon_{z z}$, where $C_{11}$ and $C_{12}$ are the elastic constants, ${ }^{54}$ and (ii) due to the $D_{2 d} \rightarrow C_{2 v}$ symmetry reduction, the only shear strain component we consider is $\epsilon_{x y}$ that describes the in-plane deformation of the (Ga,Mn)As lattice.

In the following, we restrict our calculations to zero temperature and zero magnetic field. According to the microscopic model, ${ }^{6,49,53,55}$ we can calculate the carrier contribution to the magnetic free energy. 
The effective anisotropy field $\mathbf{B}_{\text {eff }}$ acting on the magnetization is equal to $-\nabla_{\mathbf{M}} E_{c}(\mathbf{M})$, where $E_{c}$ is the carrier energy density, therefore, $E_{m}=E_{c}$. Using Eq. (B2) of Ref. 49 , we evaluate $E_{c}$ by integrating the Fermi energy with respect to the hole concentration for different magnetization orientations. Note that the main contribution of $E_{c}$ to the magnetic anisotropy comes from the spin-orbit coupling in the valence band. Once $E_{c}$ is calculated numerically as a function of the orientation of the magnetization with respect to the crystalline orientations $(\theta, \phi)$, we compare it with the phenomenological $E_{m}$ [Eq. (1)], excluding shape anisotropy, and deduce the magnetic anisotropy constants.

\section{Uniaxial anisotropy constants without and with shear strain}

In Fig. 2(a), $K_{2 \perp}$ is plotted as a function of the hole concentration, $p$, for various exchange splittings of the valence subbands, $B_{g}=\frac{A_{F} \beta_{p d} M_{s}}{6 g \mu_{B}}\left(A_{F}\right.$ is the Fermi-liquid parameter), namely, $B_{g}=-20,-30$, and $-40 \mathrm{meV}$. Setting aside the shape anisotropy, the sign change of $K_{2 \perp}$ is a signature of the change of the magnetic configuration from the $(001)$ easy-plane $\left(K_{2 \perp}<0\right)$ to the [001] easy axis $\left(K_{2 \perp}>0\right)$. The dashed line represents the experimental value of $K_{2 \perp}$ for sample A, i.e., $K_{2 \perp}=-8.5 \mathrm{~kJ} \mathrm{~m}^{-3}$, which is in good agreement with the microscopic model prediction, $-8.81 \mathrm{~kJ} \mathrm{~m}^{-3}$ when $B_{g}=-40 \mathrm{meV}$ and $p=3.5 \times 10^{20} \mathrm{~cm}^{-3}$. Adopting $B_{g}=-40 \mathrm{meV}$, one estimates $M_{s} \simeq 68 \mathrm{kA} \mathrm{m}^{-1}$, while the experimental value of the saturation magnetization for sample $\mathrm{A}$ is $M_{s} \simeq 51.3 \mathrm{kA} \mathrm{m}^{-1}$. This discrepancy might originate from the experimental difficulty in determining $M_{s}$, giving some error bar. Moreover, the adopted values of $\beta_{p d}$ and $A_{F}$ can also explain such discrepancy. Once we have validated the $\left(B_{g}\right.$, colon $\left.p\right)$ values, we focus on the shear strain effect on the uniaxial anisotropy constants. This is summarized in Fig. 2(b) which shows $K_{2 \perp}$ (black line) and $K_{2 \|}$ (red line) as a function of the $\epsilon_{x y}$ strain. The linear dependence of $K_{2 \perp}$ and $K_{2 \|}$ is clearly seen. Such behavior was expected because $K_{2 \|}$ is roughly proportional to $K_{2 \perp}$ as seen in Fig. 1. Considering alternatively the perturbation of the band structure brought by anisotropically distributed Mn dimers, Birowska ${ }^{23}$ predicts instead that $K_{2 \perp}$ is almost unchanged for a wide range of $\epsilon_{x y}$ while observing a linear behavior for $K_{2 \|}$, contradicting the experimental trend observed in the compressive regime (Fig. 1). As shown in Fig. 2(b), and for $\epsilon_{x y}=10^{-4}$, one gets $K_{2 \perp}=-8.2 \mathrm{~kJ} \mathrm{~m}^{-3}$ and $K_{2 \|}=0.96 \mathrm{~kJ} \mathrm{~m}^{-3}$, which are in good agreement with the FMR data of sample A, namely, $K_{2 \perp}=-8.5 \mathrm{~kJ} \mathrm{~m}^{-3}$ and $K_{2 \|}=1 \mathrm{~kJ} \mathrm{~m}^{-3}$. The inset of Fig. 2(b) which shows $K_{2 \|}$ as a function of $p$ confirms the same trend concerning this agreement between our theoretical predictions and the FMR data. The present $\epsilon_{x y}$ value is in line with previous estimations following a similar approach, which range from $10^{-4}$ to $5 \times 10^{-4}{ }^{6,18}$

\section{Magnetoelasticity and anisotropy constants}

Having evaluated numerically the shear strain needed to yield the experimental $K_{2 \perp}$ and $K_{2 \|}$, we now link the phenomenological constants of magnetic anisotropy with the magnetoelastic constants $\left(B_{1}, B_{2}\right)$. For this purpose, we consider the phenomenological description of the magnetoelastic energy, $E_{m e}$, which describes the
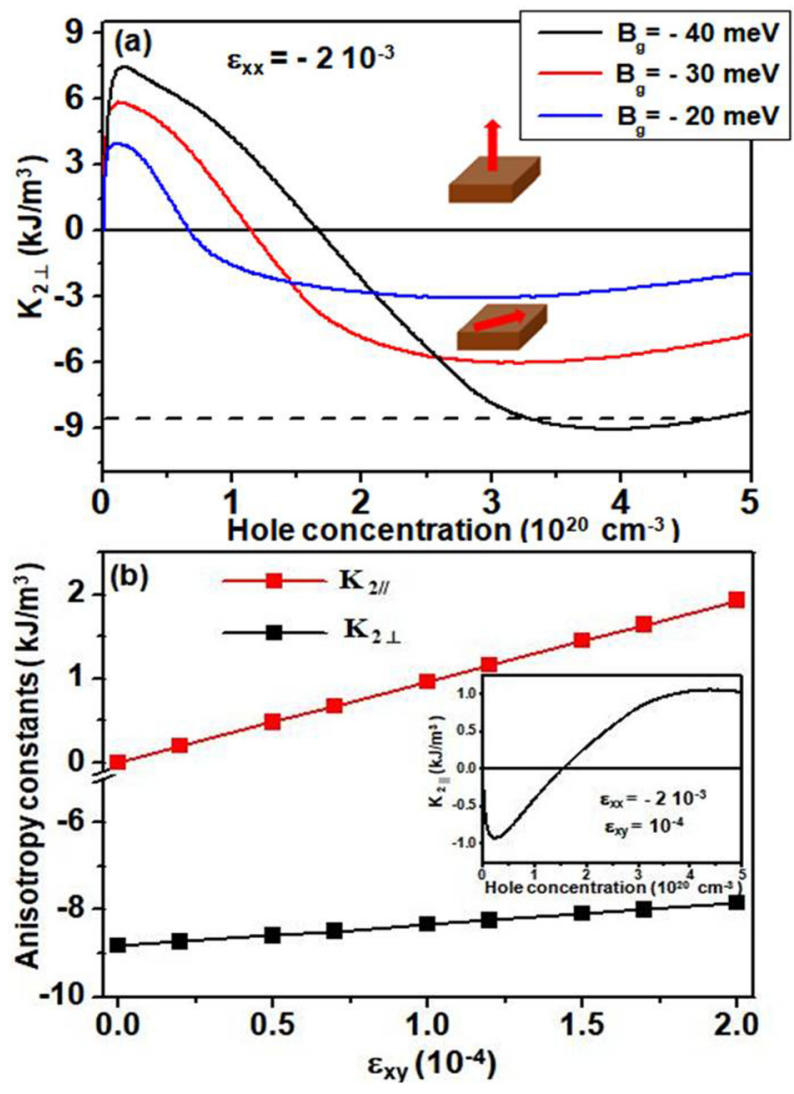

FIG. 2. (a) Perpendicular anisotropy constant $K_{2 \perp}$ as a function of the carrier density for different values of the exchange parameter $B_{g}$. The dashed line corresponds to the experimental value. (b) Perpendicular uniaxial anisotropy constant $K_{2 \perp}$, and in-plane uniaxial anisotropy constant $K_{2 \|}$ as a function of the shear strain for a carrier density $p=3.5 \times 10^{20} \mathrm{~cm}^{-3}$, with $B_{g}=-40 \mathrm{meV}$ and $\epsilon_{x x}=-2 \times 10^{-3}$. The inset shows $K_{2 \|}$ as a function of the hole concentration with $\epsilon_{x x}=-2 \times 10^{-3}$ and $\epsilon_{x y}=10^{-4}$.

interaction between the magnetization and the mechanical strain of the lattice. We start from the general expression of $E_{m e}$ in the cubic symmetry case, keeping only terms up to the second order in the magnetization components, ${ }^{56}$

$$
E_{m e}=B_{1} \epsilon_{i i}\left(m_{i}^{2}-\frac{1}{3}\right)+B_{2} \epsilon_{i j} m_{i} m_{j}
$$

Without applied magnetic field, the $E_{m e}$ energy coincides with the uniaxial components of the magnetic free energy, $E_{m}$, explicitly given by Eq. (1). Identifying Eq. (8) with Eq. (1), one can deduce

$$
K_{2 \|}=2 B_{2} \epsilon_{x y}=-6 C_{44} \lambda_{111} \epsilon_{x y}
$$




$$
\begin{aligned}
K_{2 \perp} & =-\frac{2 C_{12}+C_{11}}{2 C_{12}} B_{1} \epsilon_{z z}+B_{2} \epsilon_{x y} \\
& =\frac{3}{2} \frac{\left(2 C_{12}+C_{11}\right)\left(C_{11}-C_{12}\right)}{2 C_{12}} \lambda_{100} \epsilon_{z z}-3 C_{44} \lambda_{111} \epsilon_{x y},
\end{aligned}
$$

where $\lambda_{100}$ and $\lambda_{111}$ are the saturation magnetostriction constants when the magnetization is aligned along the [100] and [111] directions, respectively. Using Eqs. (9) and (10), we get $\lambda_{100} \simeq-27 \mathrm{ppm}$ and $\lambda_{111} \simeq-26 \mathrm{ppm}$, comparable to values for ferromagnetic magnetostrictive metals such as $\mathrm{Ni}\left(\lambda_{100} \simeq-46 \mathrm{ppm}, \lambda_{111} \simeq-24 \mathrm{ppm}\right)^{57}$ and $\mathrm{Fe}\left(\lambda_{100} \simeq 20 \mathrm{ppm}, \lambda_{111} \simeq-21 \mathrm{ppm}\right) .{ }^{57}$ These values are about 3 times larger than the ones determined experimentally (8-11 ppm), however on an unannealed sample with a low Curie temperature (57 K). ${ }^{58}$ We can also deduce $\lambda_{100} \simeq \lambda_{111}$ indicating a quasi-isotropic magnetostriction for the (Ga, Mn)As sample. Note that these $\lambda$ values give the order of magnitude of the magnetization-induced strain that would appear below the Curie temperature, which is much smaller that the shear strain discussed in this paper and would moreover depend on the magnetization orientation.

\section{SEARCHING FOR A REAL SHEAR STRAIN}

Having estimated the shear strain required to yield the observed anisotropy, we then search for its experimental evidence using either x-ray diffraction or the photoelastic effect.

\section{X-ray diffraction}

Two strategies requiring different configurations were adopted but conveying both the idea that the IP (Ga,Mn)As lattice has slightly relaxed and become rectangular in the $\langle 110\rangle$ base [Figs. 3(a) and 4(a)]. In the (C1) configuration [Fig. 3(a)], we look for [110] and [1-10] vectors of different lengths (as done in Refs. 15 and 25). For this, we perform the incident angle $(\omega$-)scan along three $90^{\circ}$-apart IP azimuths. The mapped regions were chosen to cover, in the same dataset, $\mathrm{x}$-ray diffraction signal originating both from the GaAs substrate and the $(\mathrm{Ga}, \mathrm{Mn})$ As layer. The shift along $Q_{x}\left(\Delta Q_{i}\right)$ between the substrate and layer peak positions is extracted from the $Q_{x}-Q_{z}$ map reconstruction for each azimuth $i$. From the estimated shear strain for sample A, a maximum of $\frac{\Delta Q_{i}}{Q_{\text {GaAs }}}-\frac{\Delta Q_{i+1}}{Q_{\text {GaAs }}} \approx 2 \times 10^{-4}$ can be expected for two successive [hh] IP azimuths, and none for diametrically opposed azimuths ( $i$ and $i+2$ ), as shown in the schematics of Fig. 3(a). A similar procedure is then performed along $\langle 100\rangle$ IP directions, for which we expect identical lattice spacing.

In the (C2) configuration [Fig. 4(a)], we look for angles between $\langle 100\rangle$ IP directions differing from $90^{\circ}$ for the $(\mathrm{Ga}, \mathrm{Mn}) \mathrm{As}$ layer, or equivalently that $\langle 100\rangle$ IP $(\mathrm{Ga}, \mathrm{Mn})$ As axes do not coincide with GaAs ones. To do so, azimuthal $(\phi)$ scans are performed around three $90^{\circ}$-spaced IP azimuths $i$, and compared to $[110] /[1-10]$ scans for which no difference of peak position is expected. Using again $\epsilon_{x y}=10^{-4}$, a maximum spacing of $\Delta \phi=\phi_{\mathrm{GaAs}, i}-\phi_{(\mathrm{Ga}, \mathrm{Mn}) \mathrm{As}, i}=(-1)^{i} 12 \mathrm{mdeg}$ can be anticipated for hol peaks: a signature of shear strain is indeed that the angle between $[100] /[010]$ vectors of $(\mathrm{Ga}, \mathrm{Mn})$ As should alternatively be smaller $\left(<90^{\circ}\right)$ or larger $\left(>90^{\circ}\right)$ than for GaAs [see schematics of Fig. 4(a)]. For $h h l$ peaks, we expect from this scenario $\Delta \phi=0$ for all azimuths.

Experiments were performed at room temperature on the DiffAbs beamline of the SOLEIL synchrotron. High resolution reciprocal space maps around the various diffraction peaks were performed. By using a hybrid pixel area detector (XPAD) covering an angular range of several degrees, these measurements are typically realized by scanning one of the sample angles/orientations around the value satisfying Bragg's law. Data are acquired during the continuous movement (rotation) of the motor (which is optically encoded) for achieving angular resolution better than $0.1 \mathrm{mdeg}$. Corresponding volumes in the reciprocal space $\left(Q_{x}, Q_{y}, Q_{z}\right.$ coordinates) were reconstructed and $\mathrm{x}$-ray diffraction peak position was extracted from $2 \mathrm{D}$ planar cuts. Indices $h, k, l$ were chosen low enough to have both peaks appearing on the $2 \mathrm{D}$ XPAD detector and $l \neq 0$, but large enough to clearly separate $\mathrm{GaAs}$ and $(\mathrm{Ga}, \mathrm{Mn})$ As peaks for $(\mathrm{C} 1)$. Although $\mathrm{X}$-ray diffraction using a well collimated x-ray beam (e.g., like the one at a synchrotron facility) is expected to detect such quantities, we should point out here the particular difficulty related to the presence of the very sharp and intense GaAs substrate diffraction peaks located extremely close to the $(\mathrm{Ga}, \mathrm{Mn})$ As epitaxial layer peaks. With indices

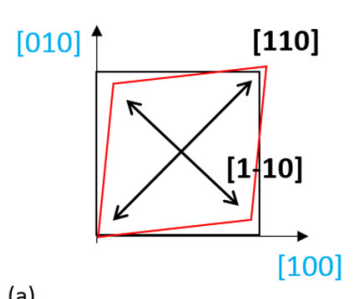

(a)

[100]

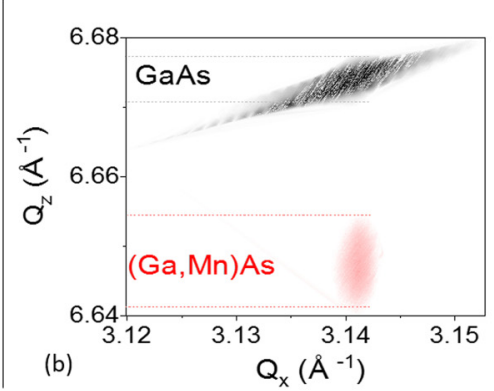

(b)

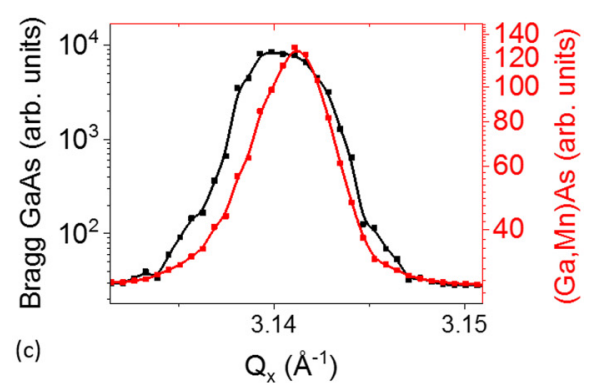

FIG. 3. (a) Schematics of a sheared in-plane (Ga,Mn)As lattice (in red) over GaAs (in black). In reciprocal space maps, we look for misalignments of substrate and layer peak position along $Q_{x}$. (b) Reconstructed $Q_{x} / Q_{z}$ map for the (226) diffraction peak (log scale). (c) Projection along the $Q_{x}$ direction of the substrate and layer diffraction peaks averaged between the dotted lines of $(b)$, evidencing a slight shift, but which is of the same order of magnitude between consecutive and opposite peaks. Experiments are performed at room temperature. 

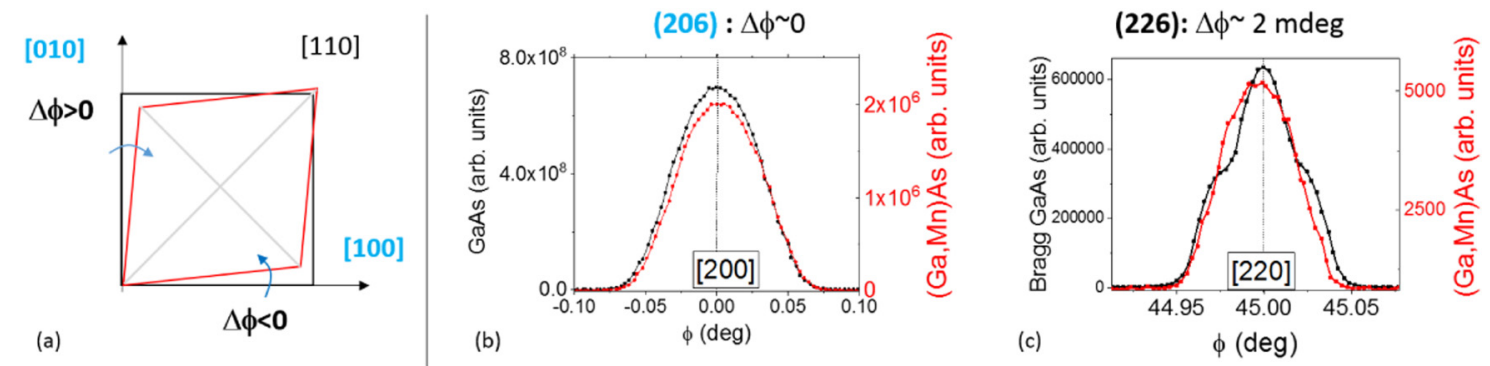

FIG. 4. (a) Schematics of sheared in-plane lattice. In $(\phi)$-scans, we look for misalignments of $\langle 100\rangle$ directions between the (Ga,Mn)As (in red) and GaAs (in black) lattice. (b) Typical $\phi$-scans for (206) planes, with $\omega=15.8238^{\circ}$ (respectively $\omega=15.6280^{\circ}$ ) for the Bragg [respectively (Ga,Mn)As] peak. (c) Typical $\phi$-scans for (226) planes, with $\omega=10.9522^{\circ}$ (respectively $\omega=10.8197^{\circ}$ ) for the GaAs [respectively $(\mathrm{Ga}, \mathrm{Mn}) \mathrm{As}$ ] peak. We define $\Delta \phi=\phi_{\mathrm{GaAs}}-\phi_{(\mathrm{Ga}, \mathrm{Mn}) \mathrm{As}}$. "Wings" appear on either side of the GaAs substrate peak, a possible signature of truncation rods. Experiments are performed at room temperature.

verifying $h+k+l=4 n+2$ for the $(h h l)$ peak, the energy was moreover tuned for anomalous diffraction in order to minimize the GaAs signal. ${ }^{59}$ The final configuration was to study (206) and (226) peaks at $E=12.35 \mathrm{keV}$. The $\mathrm{x}$-ray beam spot size was around $150 \mu \mathrm{m}$. Although anomalous diffraction conditions were fulfilled for (226) planes, the Bragg peak intensity remained over 100 times larger than that of the $45 \mathrm{~nm}$ thick magnetic layer, because of the much deeper penetration depth of $\mathrm{x}$-rays (about $2 \mu \mathrm{m}$ ).

Typical maps and cuts realized in the $(\mathrm{C} 1)$ configuration are shown in Figs. 3(b) and 3(c). A systematic difference of IP peak position was observed between the substrate and the sample A layer, corresponding to $\frac{\Delta Q_{i}}{Q_{\text {GaAs }}}-\frac{\Delta Q_{i+1}}{Q_{\text {GaAs }}} \approx-2 \pm 0.5 \times 10^{-4}$ for (226) peaks and $1.2 \pm 0.5 \times 10^{-4}$ for (206) peaks. Fairly large deviations were observed between repeated measurements; however, no significant difference was observed between 2 consecutive or opposite peaks, contrary to what is expected from shear strain. As a result, we could at best conclude on a very marginal isotropic IP relaxation of the layer, but not on any nonequivalent lattice spacing along $[110] /[1-10]$ directions as opposed to [100]/[010] ones. Using these data, we also searched inconclusively for signatures of anisotropic coherence length [i.e., anisotropic (226) layer peak widths], and anisotropic structure factor [i.e., anisotropic ratio of integrated intensity of $\mathrm{GaAs}$ and $(\mathrm{Ga}, \mathrm{Mn})$ As peaks].

Typical results obtained in the (C2) configuration are shown in Figs. 4(b) and 4(c). A clear and reproducible angular $\Delta \phi=-2 \pm 0.3$ mdeg was observed for the (226) direction. This (226) misalignment had the same sign for two consecutive azimuths, which should indicate a bulk rotation of the layer IP lattice with respect to the substrate, rather than a shearing. However, no misalignment was observed on (206) peaks (within the standard deviation) to validate this bulk rotation. As for the (C1) configuration, no robust evidence of a shear strain could be found in this high IP anisotropy layer.

\section{Photoelastic effect}

We turned to another method with a potentially larger sensitivity to strain: the photoelastic effect.

Owing to the photoelastic effect, the dielectric permittivity tensor of a zinc blende crystal is modified. ${ }^{60}$ The biaxial strain does not induce any birefringence but an $\epsilon_{x y}$ shear strain introduces an off-diagonal matrix element that produces different refractive indices for linear polarization along the [110] and [-110] crystallographic axes. Consequently, for near-normal incidence, a linear polarization making an angle $\beta$ with the [110] direction will rotate by an angle $\Delta \beta_{p e}=\Delta \beta_{p e}^{0} \sin (2 \beta)$ with

$$
\Delta \beta_{p e}^{0}=\operatorname{Re}\left[\frac{C_{44} P_{44} \epsilon_{x y}\left(1-\exp \left(i \frac{4 \pi n t}{\lambda}\right)\right)}{n\left(n^{2}-1\right)}\right],
$$

where $n$ is the complex refractive index [we assume the same index for the $(\mathrm{Ga}, \mathrm{Mn})$ As layer and the GaAs substrate], $t$ is the layer thickness, $C_{44}$ is the elastic constant, and $P_{44}=4 P_{1212}, P$ being the stress-optical tensor. ${ }^{61,62}$ It is this exact term that was used for the static or time-resolved detection of SAW-induced variations of strain in Refs. 63 and 27. Taking the experimental values for $P_{44}$ and $n$ from Ref. 61 at the optical wavelength $\lambda=532 \mathrm{~nm}$ and $\epsilon_{x y}=10^{-4}$ we estimate $\Delta \beta_{p e}^{0} \approx 15 \mathrm{mdeg}$, which should be easily detected using a photoelastic modulator. ${ }^{64}$ Once again, if it is a real shear strain that induces the IP anisotropy, it should persist at room temperature.

Four samples were investigated: Sample A with strong IP uniaxial anisotropy, sample B with biaxial anisotropy, an epiready GaAs substrate, and a $100 \mathrm{~nm}$ aluminum layer evaporated on a glass substrate.

The polarization rotation was measured at room temperature in the reflection geometry as a function of the angle between an IP crystallographic axis (or an arbitrary axis in the case of Al/glass) and the incident linear polarization using a photoelastic modulator. In order to rotate the sample in its plane without any change in the path of the reflected light, the sample was mounted on an x-ray goniometer. The sample plane was perpendicular to the plane of incidence. The angle of incidence was $19^{\circ}$. The $532 \mathrm{~nm}$-laser beam was linearly polarized perpendicular to the plane of incidence. The reflected beam was modulated by a photoelastic modulator (frequency $f=42 \mathrm{kHz}$ ) with axes parallel and perpendicular to the incident polarization before passing through an analyser set at $45^{\circ}$ to the axes and being detected by a photodiode. The Kerr rotation signal was detected at $2 f$ by a lock-in amplifier. The reflected signal 


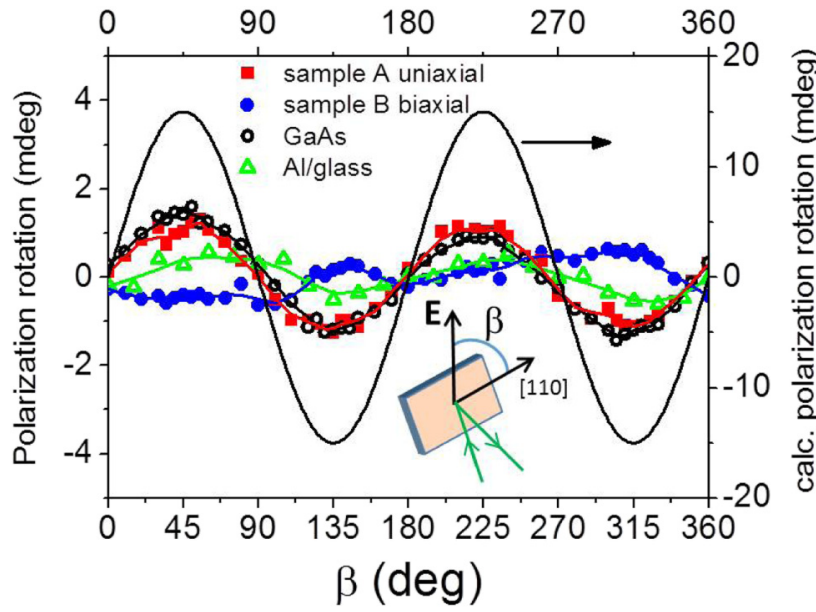

FIG. 5. Polarization rotation angle $\Delta \beta$ (left scale) as a function of $\beta$, the angle of rotation of the sample in its plane, for a fixed vertical light polarization $E$. For Sample A, Sample B, and the GaAs substrate, $\beta$ is counted from the [110] axis as indicated in the inset. Right scale: the polarization rotation expected from the photoelastic effect assuming $\epsilon_{x y}=10^{-4}$.

at the frequency of a mechanical chopper was used to normalize the signal.

Figure 5 shows the polarization rotation for the four samples. For the Al/glass sample (triangles), there is hardly any dependence on the rotation angle, likewise for sample B with biaxial anisotropy (full circles). Sample A, with uniaxial anisotropy (squares), shows a $\sin (2 \beta)$ dependence, indeed related to the crystallographic axes as we have checked, but with an amplitude of less than $1.5 \mathrm{mdeg}$, i.e., ten times smaller than expected for $\epsilon_{x y}=10^{-4}$. This places an upper boundary for a genuine shear strain in the layer of $\epsilon_{x y}=10^{-5}$, just below our x-ray detection threshold. However, this polarization rotation is probably related to GaAs, since it is the same on sample A and the bare substrate (empty circles). Since there is no such polarization rotation on sample B, this might depend on the quality of the substrate.

Therefore, we conclude that the expected shear strain is not found although well within the detectability range of the optical measurements.

\section{DISCUSSION AND CONCLUSION}

We looked for experimental evidence of a structural shear strain being at the roots of strong in-plane magnetic anisotropy in (Ga,Mn) As, relying on $\mathrm{x}$-ray diffraction or photoelastic effect-induced optical polarization rotation. While the search for anisotropic [110] and $[1-10]$ vector lengths proved inconclusive due to poor experimental reproducibility, that of $[100] /[010]$ angles differing from $90^{\circ}$ clearly ruled out the existence of a physical $\epsilon_{x y}=10^{-4}$ strain compatible with the $\mathbf{k} \cdot \mathbf{p}$ theory estimations of our anisotropy. This was confirmed by photoelastic effect measurements. We thus conclude on the absence of a physical shear strain responsible for the observed uniaxial anisotropy, and instead on a mechanism capable of being modeled as an effective shear strain. Paralleling the preferential attachment of $\mathrm{Fe}$ atoms to As-rich planes leading to strong uniaxial uniaxial anisotropy observed on $\mathrm{Fe} / \mathrm{GaAs},{ }^{12}$ one can invoke the possible anisotropic incorporation of $\mathrm{Mn}$ atoms, ${ }^{25}$ or $\mathrm{Mn}$ dimer formation $^{13,23}$ when growing $(\mathrm{Ga}, \mathrm{Mn}) \mathrm{As}$ on a GaAs substrate. These would be equivalent to a shear strain in a hypothetical free $(\mathrm{Ga}, \mathrm{Mn})$ As layer, thereby inducing magnetic anisotropy as the theory developed above shows, but be undetectable once epitaxied perfectly onto the substrate. Quite challenging to observe directly by microscopy techniques, such an anisotropic magnetic atom distribution might, however, show up as a measurable anisotropy of the exchange constant (at low temperatures). A positive conclusion to such an experiment would help to devise growth strategies capable of tuning this magnetic anisotropy. Independent of the origins of this effective shear strain, our comparison of data taken on a large number of articles highlights that the surest way of obtaining a strong IP anisotropy in $(\mathrm{Ga}, \mathrm{Mn}) \mathrm{As}$ remains to target a large out-of-plane anisotropy, often the result of a combination of large lattice mismatch, optimized anneal, and high effective Mn concentration.

\section{ACKNOWLEDGMENTS}

This work was financially supported by the Tunisian Ministry of Higher Education and Scientific Research and by the French Ministry of Foreign Affairs through the project PHC Utique (No. 16G1303). We acknowledge the SOLEIL Synchrotron for providing the beamtime for the diffraction experiments (proposal No. 20180469). We thank H. J. von Bardeleben from INSP for the FMR data and F. Vidal and M. Vabre from INSP for providing and fixing the $\mathrm{x}$-ray goniometer for optical experiments.

\section{REFERENCES}

${ }^{\mathbf{1}}$ S. Iwasaki, IEEE Trans. Magn. 16, 71 (1980).

${ }^{2}$ L. Thevenard, B. Boutigny, N. Güsken, L. Becerra, C. Ulysse, S. Shihab, A. Lemaître, J.-V. Kim, V. Jeudy, and C. Gourdon, Phys. Rev. B 95, 054422 (2017).

${ }^{3}$ S. Davis, J. A. Borchers, B. B. Maranville, and S. Adenwalla, J. Appl. Phys. 117, 063904 (2015).

${ }^{4}$ I. Camara, J.-Y. Duquesne, A. Lemaitre, C. Gourdon, and L. Thevenard, Phys. Rev. Appl. 11, 014045 (2019).

${ }^{5}$ D. Givord, O. F. K. McGrath, C. Meyer, and J. Rothman, J. Magn. Magn. Mater. 157-158, 245 (1996).

6. Zemen, J. Kučera, K. Olejník, and T. Jungwirth, Phys. Rev. B 80, 155203 (2009).

7J. Li and P. M. Haney, Appl. Phys. Lett. 109, 032405 (2016).

${ }^{8}$ O. Thomas, Q. Shen, P. Schieffer, N. Tournerie, and B. Lépine, Phys. Rev. Lett. 90, 017205 (2003).

${ }^{9}$ S. Zhou, L. Chen, A. Shalimov, J. Zhao, and M. Helm, AIP Adv. 2, 042102 (2012).

${ }^{10}$ E. M. Kneedler, B. T. Jonker, P. M. Thibado, R. J. Wagner, B. V. Shanabrook, and L. J. Whitman, Phys. Rev. B 56, 8163 (1997).

${ }^{11}$ M. Zölfl, M. Brockmann, M. Köhler, S. Kreuzer, T. Schweinböck, S. Miethaner, F. Bensch, and G. Bayreuther, J. Magn. Magn. Mater. 175, 16 (1997).

${ }^{12}$ K. Tivakornsasithorn, X. Liu, X. Li, M. Dobrowolska, and J. K. Furdyna, J. Appl. Phys. 116, 043915 (2014).

${ }^{13}$ M. Birowska, C. Śliwa, J. A. Majewski, and T. Dietl, Phys. Rev. Lett. 108, 237203 (2012).

${ }^{\mathbf{1 4}}$ Y. Hashimoto, Y. Iye, and S. Katsumoto, J. Cryst. Growth 378, 381 (2013). 
${ }^{15} \mathrm{U}$. Welp, V. K. Vlasko-Vlasov, A. Menzel, H. D. You, X. Liu, J. K. Furdyna, and T. Wojtowicz, Appl. Phys. Lett. 85, 260 (2004).

${ }^{16}$ J. Wunderlich, A. C. Irvine, J. Zemen, V. Holý, A. W. Rushforth, E. De Ranieri, U. Rana, K. Výborný, J. Sinova, C. T. Foxon, R. P. Campion, D. A. Williams, B. L. Gallagher, and T. Jungwirth, Phys. Rev. B 76, 054424 (2007).

17. Shiogai, D. Schuh, W. Wegscheider, M. Kohda, J. Nitta, and D. Weiss, Appl. Phys. Lett. 98, 98 (2011).

${ }^{18}$ M. Sawicki, K.-Y. Wang, K. W. Edmonds, R. P. Campion, C. R. Staddon, N. R. S. Farley, C. T. Foxon, E. Papis, E. Kamińska, A. Piotrowska, T. Dietl, and B. L. Gallagher, Phys. Rev. B 71, 121302 (2005).

${ }^{19}$ S. Piano, X. Marti, A. W. Rushforth, K. W. Edmonds, R. P. Campion, M. Wang, O. Caha, T. U. Schülli, V. Holý, and B. L. Gallagher, Appl. Phys. Lett. 98, 152503 (2011).

${ }^{20}$ S. van Dijken, G. Di Santo, and B. Poelsema, Appl. Phys. Lett. 77, 2030 (2000).

${ }^{\mathbf{2 1}}$ A. Muñoz-Noval, E. Salas-Colera, and R. Ranchal, J. Phys. Chem. C 123, 13131 (2019).

${ }^{22}$ A. W. Rushforth, E. De Ranieri, J. Zemen, J. Wunderlich, K. W. Edmonds, C. S. King, E. Ahmad, R. P. Campion, C. T. Foxon, B. L. Gallagher, K. Výborný, J. Kučera, and T. Jungwirth, Phys. Rev. B 78, 085314 (2008).

${ }^{\mathbf{2 3}}$ M. Birowska, J. Magn. Magn. Mater. 432, 396 (2017).

${ }^{24}$ H. Subramanian and J. E. Han, J. Phys. Condens. Matter 25, 206005 (2013).

${ }^{25}$ M. Kopecký, J. Kub, F. Máca, J. Mažek, O. Pacherová, A. W. Rushforth, B. L. Gallagher, R. P. Campion, V. Novák, and T. Jungwirth, Phys. Rev. B 83, 235324 (2011).

${ }^{26}$ P. Kuszewski, I. S. Camara, N. Biarrotte, L. Becerra, J. von Bardeleben, W. Savero Torres, A. Lemaitre, C. Gourdon, J.-Y. Duquesne, and L. Thevenard, J. Phys. Condens. Matter 30, 244003 (2018)

${ }^{27}$ P. Kuszewski, J.-Y. Duquesne, L. Becerra, A. Lemaître, S. Vincent, S. Majrab, F. Margaillan, C. Gourdon, and L. Thevenard, Phys. Rev. Appl. 10, 034036 (2018).

${ }^{\mathbf{2 8}}$ D. K. Biegelsen, R. D. Bringans, J. E. Northrup, and L.-E. Swartz, Phys. Rev. B 41, 5701 (1990).

${ }^{29}$ Q. Xue, T. Hashizume, J. M. Zhou, T. Sakata, T. Ohno, and T. Sakurai, Phys. Rev. Lett. 74, 3177 (1995).

${ }^{30}$ M. S. Brandt, S. T. B. Goennenwein, T. A. Wassner, F. Kohl, A. Lehner, H. Huebl, T. Graf, M. Stutzmann, A. Koeder, W. Schoch, and A. Waag, Appl. Phys. Lett. 84, 2277 (2004).

${ }^{31}$ S. T. B. Goennenwein, T. Graf, T. Wassner, M. S. Brandt, M. Stutzmann, J. B. Philipp, R. Gross, M. Krieger, K. Zurn, P. Ziemann, A. Koeder, S. Frank, W. Schoch, and A. Waag, Appl. Phys. Lett. 82, 730 (2003).

${ }^{32}$ P. Němec, V. Novák, N. Tesařová, E. Rozkotová, H. Reichlová, D. Butkovičová, F. Trojánek, K. Olejník, P. Malý, R. Campion, B. Gallagher, J. Sinova, and T. Jungwirth, Nat. Commun. 4, 1422 (2013).

${ }^{33}$ A. Chernyshov, M. Overby, X. Liu, J. K. Furdyna, Y. Lyanda-Geller, and L. P. Rokhinson, Nat. Phys. 5, 656 (2009).

${ }^{34}$ D. Fang, H. Kurebayashi, J. Wunderlich, K. Výborný, L. P. Zârbo, R. P. Campion, A. Casiraghi, B. L. Gallagher, T. Jungwirth, and A. J. Ferguson, Nat. Nanotechnol. 6, 413 (2011).

${ }^{35}$ V. K. Vlasko-Vlasov, W. K. Kwok, S. Dong, X. Liu, M. Dobrowolska, and J. K. Furdyna, Phys. Rev. B 98, 180411 (2018).

${ }^{36} \mathrm{~K}$. Khazen, H. von Bardeleben, J. Cantin, L. Thevenard, L. Largeau, O. Mauguin, and A. Lemaître, Phys. Rev. B 77, 165204 (2008).

${ }^{37}$ Using Fig. 4 of Ref. 32, we calculated their $K_{2 \|}$ and $K_{2 \perp}$ using $K_{\text {out }}(m T)=B_{2 \perp}-\mu_{0} M_{s} / 2$ and assumed that all Mn were magnetically active to convert their anisotropy fields $\left(K_{\text {out }}, K_{u}\right)$ into anisotropy constants.
${ }^{38}$ S. Haghgoo, M. Cubukcu, H. J. von Bardeleben, L. Thevenard, A. Lemaitre, and C. Gourdon, Phys. Rev. B 82, 041301(R) (2010).

${ }^{39}$ L. Thevenard, C. Gourdon, S. Haghgoo, J.-P. Adam, H. J. von Bardeleben, A. Lemaître, W. Schoch, and A. Thiaville, Phys. Rev. B 83, 245211 (2011)

${ }^{40}$ L. Thevenard, E. Peronne, C. Gourdon, C. Testelin, M. Cubukcu, E. Charron, S. Vincent, A. Lemaître, and B. Perrin, Phys. Rev. B 82, 104422 (2010).

${ }^{41}$ M. Cubukcu, H. J. von Bardeleben, K. Khazen, J. L. Cantin, O. Mauguin, L. Largeau, and A. Lemaître, Phys. Rev. B 81, 041202 (2010).

${ }^{42}$ H. Riahi, W. Ouerghui, L. Thevenard, C. Gourdon, M. Maaref, A. Lemaître, O. Mauguin, and C. Testelin, J. Magn. Magn. Mater. 342, 149 (2013).

${ }^{43}$ S. Shihab, H. Riahi, L. Thevenard, H. J. von Bardeleben, A. Lemaittre, and C. Gourdon, Appl. Phys. Lett. 106, 142408 (2015).

${ }^{44}$ F. Matsukura and H. Ohno, Jpn. J. Appl. Phys. 54, 098003 (2015).

${ }^{45}$ J. M. Luttinger and W. Kohn, Phys. Rev. 97, 869 (1955).

${ }^{46} \mathrm{M}$. Yahyaoui, K. Boujdaria, M. Cubukcu, C. Testelin, and C. Gourdon, J. Phys. Condens. Matter 25, 346001 (2013).

${ }^{47}$ O. Madelung, U. Rossler, and M. Schulz, Landolt-Bornstein, New Series, Group III, Vol. 41 (Berlin, Springer-Verlag, 2005).

${ }^{48}$ T. Dietl, H. Ohno, F. Matsukura, J. Cibert, and e. D. Ferrand, Science 287, 1019 (2000).

${ }^{49}$ T. Dietl, H. Ohno, and F. Matsukura, Phys. Rev. B 63, 195205 (2001).

${ }^{50} \mathrm{G}$. Pikus and G. Bir, Tverd. Tela (Leningrad) 1, 1642 (1960).

${ }^{51}$ G. G. Koster, J. Dimmock, R. G. Wheeler, and H. Statz, Properties of the Thirty-Two Point Groups (MIT Press, 1963), Vol. 24.

${ }^{52}$ The basis wave functions used for the matrix representation of $\mathcal{H}_{S}$ are extracted from Table 83, p. 94, of Koster et al..$^{51}$ representing the direct product of two irreducible representations, namely, $\Gamma_{5} \otimes \Gamma_{6}=\Gamma_{7}+\Gamma_{8}$. In this Table, $\left(u_{y z}^{5}, u_{x z}^{5}, u_{x y}^{5}\right)$ indicate $(X, Y, Z) \mathrm{p}$ orbitals, $v_{1 / 2}^{6}=\uparrow$ and $v_{-1 / 2}^{6}=\downarrow$ where $\uparrow$ $(\downarrow)$ indicates spin up (spin down) states. $\psi_{-1 / 2}^{7}=\left|\frac{1}{2},-\frac{1}{2}\right\rangle$ and $\psi_{1 / 2}^{7}=\left|\frac{1}{2}, \frac{1}{2}\right\rangle$ represent the usual split-off valence bands (VBs), $\psi_{-3 / 2}^{8}=\left|\frac{3}{2}, \frac{1}{2}\right\rangle$ and $\psi_{3 / 2}^{8}=$ $\left|\frac{3}{2},-\frac{1}{2}\right\rangle$ indicate the light-hole VBs, $\psi_{-1 / 2}^{8}=\left|\frac{3}{2}, \frac{3}{2}\right\rangle$ and $\psi_{1 / 2}^{8}=\left|\frac{3}{2}, \quad-\frac{3}{2}\right\rangle$ represent the heavy-hole VBs.

${ }^{53}$ M. Glunk, J. Daeubler, L. Dreher, S. Schwaiger, W. Schoch, R. Sauer, W. Limmer, A. Brandlmaier, S. Goennenwein, C. Bihler et al., Phys. Rev. B 79, 195206 (2009).

${ }^{54}$ I. Vurgaftman, J. Á. Meyer, and L. Á. Ram-Mohan, J. Appl. Phys. 89, 5815 (2001).

${ }^{55} \mathrm{M}$. Yahyaoui, C. Testelin, C. Gourdon, and K. Boujdaria, J. Appl. Phys. 111, 033902 (2012)

${ }^{56}$ Magnetism I- Fundamentals, edited by E. du Trémolet de Lacheisserie D. Gignoux, and M. Schlenker (Kluwer Academic Publishers, 2003).

${ }^{57}$ R. Grössinger, R. S. Turtelli, and N. Mehmood, in IOP Conference Series: Materials Science and Engineering (IOP Publishing, 2014), Vol. 60, p. 012002.

${ }^{58}$ S. C. Masmanidis, H. X. Tang, E. B. Myers, M. Li, K. De Greve, G. Vermeulen, W. Van Roy, and M. L. Roukes, Phys. Rev. Lett. 95, 187206 (2005).

${ }^{59}$ T. U. Schülli, M. Sztucki, V. Chamard, T. H. Metzger, and D. Schuh, Appl. Phys. Lett. 81, 448 (2002)

${ }^{60}$ D. Royer and E. Dieulesaint, Elastic Waves in Solids (Springer-Verlag, Heidelberg, 2001).

${ }^{61}$ P. Etchegoin, J. Kircher, M. Cardona, C. Grein, and E. Bustarret, Phys. Rev. B 46, 15139 (1992).

${ }^{62}$ M. Cardona, D. Rönnow, and P. V. Santos, Thin Solid Films 313, 10 (1998).

${ }^{63}$ P. V. Santos, Appl. Phys. Lett. 74, 4002 (1999).

${ }^{64}$ K. Sato, Jpn. J. Appl. Phys. 20, 2403 (1981). 